\title{
DISTRIBUTION OF GAMMA-RAY BURSTS IN HALO NEUTRON STAR-COMET MODELS
}

\author{
M.G. HIGGINS and R.N. HENRIKSEN \\ Queen's University at Kingston \\ Kingston, Ontario, Canada
}

\begin{abstract}
.
The motions of comets and neutron stars have been integrated over five billion years in the Galactic potential to determine a gamma-ray burst distribution, presuming that bursts are the result of interactions between these two families of objects. The comets originate in two distinct populations - one from ejection by stars in the Galactic disk, and the other from ejection by stars in globular clusters. No choice of the free parameters resulted in agreement with both the isotropy data and the $\log (N>F)-\log (F)$ data.
\end{abstract}

Key words: Gamma-ray bursts - Neutron stars - Comets - Globular clusters

\section{Introduction}

There have been many models over the years which try to identify gammaray bursts (GRBs) with neutron stars (NSs) in an extended Halo around the Galaxy which interact in some way with passing comets (e.g. Pineault and Duquet (1993) or Mitrofanov and Sagdeev (1990)). The details of the environment which would allow such a scenario involving interstellar comets have not been worked out in detail.

Over the age of the solar system, it has been estimated that approximately $10^{12}$ comets have escaped the Sun's gravitational well (Stern (1990)). If other solar systems behave in a similar manner, there should be a large number of free comets in globular clusters (GCs), which, after escaping the clusters by scattering, could seed an extended Halo.

This work evolved a population of $10^{6} \mathrm{NSs}$ born in the disk. It also evolved $10^{7}$ comets ejected from GCs, and assumed a population of disk comets which follow the mass density of the disk. Using these results, a sample of GRBs was created.

\section{The Model of Galactic Potential}

The model used was taken from Paczynski (1990), with some modifications. It assumes three contributions to the total potential: a disk, a spheroid, and a spherical dark matter halo. The values used for the constants in his model, relating to the oblateness of the spheroid $\left(a_{1}\right.$ and $\left.b_{1}\right)$ and disk $\left(a_{2}\right.$ and $\left.b_{2}\right)$, are: $a_{1}=0, b_{1}=0.277 \mathrm{kpc}, a_{2}=3.7 \mathrm{kpc}$, and $b_{2}=0.2 \mathrm{kpc}$. A dark matter halo with constant density inside a radius $r_{\mathrm{c}}=8 \mathrm{kpc}$, and density falling off like $r^{-2.25}$ outside $r_{\mathrm{c}}$, replaced Paczynski's halo. The masses used are: 
$\mathrm{M}_{1}=1.12 \times 10^{10} \mathrm{M}_{\odot}($ the spheroid mass $), \mathrm{M}_{2}=8.07 \times 10^{10} \mathrm{M}_{\odot}$ (the disk mass), $\mathrm{M}_{\mathrm{c}}=1.75 \times 10^{10} \mathrm{M}_{\odot}$ (the dark halo mass inside $r_{\mathrm{c}}$ ).

Other components contributing to the Galactic potential, such as satellite galaxies or the globular cluster population, were ignored for simplicity. Over the course of the simulation, roughly $10 \%$ of the comets would be affected by such objects; this is not expected to change the results to any great degree. In addition, it is assumed that the Galactic dark matter does not interact with the comets to any appreciable degree. This assumption would fail if the dark matter is mainly molecular clouds, for example.

\section{The Three Populations}

The GC population comets were ejected from the GC stellar systems with small relative speeds. A small fraction of these comets escaped from the GC without scattering; however, a much larger fraction were scattered by stars in the GC (for simplicity, all scattering stars were taken to have a mass $\mathrm{M}_{\odot}$ ), and were ejected from the GC with speeds approximately $10-20 \mathrm{~km} / \mathrm{s}$. 140 typical GCs were used in the simulation. The GC population was found by assuming a radial distribution which falls off like $r^{-0.85}$ (Kulessa and Lynden-Bell (1992)), and a velocity distribution with gaussian form, with average value $v_{0}=220-2 r_{\mathrm{kpc}} \mathrm{km} / \mathrm{s}$, and standard deviation $\sigma=120-r_{\mathrm{kpc}}$ $\mathrm{km} / \mathrm{s}$. Here, $r_{\mathrm{kpc}}$ is the radial distance from the centre of the Galaxy in kpc, allowed to range from $6-110 \mathrm{kpc}$. Each GC was taken to have mass $10^{5} \mathrm{M}_{\odot}$, and the Plummer model, with a radial distance scale of $1 \mathrm{pc}$, was used for the gravitational potential.

The disk population of comets was taken to follow the disk component of the total density. The total number of disk comets was set to the total number of GC comets multiplied by the ratio of the disk mass to the total mass of GCs, and a factor $f$ (one of the free parameters in the work), which is a ratio of the efficiencies of comet ejection in the disk to comet ejection in the GCs.

The NSs were started in the disk, with a position distribution which follows the observed pulsar distribution, taken from Johnston (1994). The initial speeds considered ranged from $350 \mathrm{~km} / \mathrm{s}$ to $2000 \mathrm{~km} / \mathrm{s}$ in a frame of reference rotating around the Galactic centre.

\section{Estimating the Interaction Probability}

The probability of interaction between the NS and comet populations was taken to be (e.g. Pineault and Poisson (1989)) $P=\frac{A n_{\text {ns }} n_{\mathrm{c}}}{v_{\text {rel }}}$. Here, $A$ is a constant relating to the cross-section for collisions, $n_{\mathrm{ns}}$ is the NS number density, normalised to unity at the maximum value, $n_{\mathrm{c}}$ is the normalised total comet number density (disk comets plus GC comets), and $v_{\text {rel }}$ is the average 
Fig. 1. Projected sky map of 5000 sample GRBs for NS birth velocities of $700 \mathrm{~km} / \mathrm{s}$, $f=0$

relative speed of the two populations at the point in question, determined in the simulations. This includes only gravitational focussing in the collisions.

The burst frequency in this model can be roughly estimated, but since much of the physics is unknown, assigning values to the constants should be taken as tentative at best. Very approximately, $\frac{d N_{\text {bursts }}}{d t}=N_{\mathrm{c}} \tilde{n}_{\mathrm{NS}} \sigma_{\text {int }} v_{\text {rel }}$, where $N_{\mathrm{c}}$ is the total number of comets, $\tilde{n}_{\mathrm{NS}}$ is an average number density of NSs, and $\sigma_{\text {int }}$ is the interaction cross-section.

The total number of comets is given by $N_{\mathrm{c}}=N_{\mathrm{sGC}} N_{\mathrm{cGC}}+N_{\mathrm{sd}} N_{\mathrm{cd}}$, where $N_{\mathrm{sGC}}$ is the total number of GC stars $\left(\simeq 10^{7}\right), N_{\mathrm{sd}}$ is the total number of disk stars $\left(\simeq 10^{10}\right), N_{\mathrm{cd}}$ is the number of comets produced by a disk star, and $N_{\text {cGC }}$ is the number of comets produced by a GC star; using the efficiency ratio $f$ from section 3, $N_{\mathrm{cGC}}=N_{\mathrm{cd}} / f$.

As a rough estimate, we can use the number of comets estimated to have been ejected from our solar system as an approximation to $N_{\text {cd }}$. Assuming a total population of $10^{9} \mathrm{NSs}$, most inside a sphere of radius $40 \mathrm{kpc}$, relative speeds on the order of $10^{3} \mathrm{~km} / \mathrm{s}$, and that the cross-section is just the square of the light-cylinder radius for a NS spinning with an angular velocity of 0.1 $\mathrm{rad} / \mathrm{s}$, the number of bursts per day is roughly $\frac{d N}{d t} \simeq 4$ bursts/day $\left(\frac{10^{-6}}{f}\right)$.

\section{Simulated Gamma-Ray Burst Distribution}

Figure 1 shows a sky map of 5000 sample GRB positions, using a NS birth velocity of $700 \mathrm{~km} / \mathrm{s}$ and efficiency ratio $f=0$ (no disk comets). The anisotropy toward the Galactic disk is obvious, even without including the effect of the disk comet population. The clustering is evident with as few as 585 bursts (as in the BATSE 2B catalog). Including a disk population rapidly (for any $f>10^{-5}$ ) makes clumping toward the Galactic plane extremely efficient. Changing the birth velocities of NSs has little effect, 
due to the clumped comet population. Note that increasing $f$ beyond $10^{-5}$ not only causes clumping toward the disk, but also decreases the number of bursts/day to numbers much smaller than are observed. The requirement of such a discrepancy in the ejection efficiencies is another strike against this model.

The flux of a burst was calculated by letting the total energy $E$ of a burst follow a gaussian distribution in logarithmic space, with the average burst energy set so that a burst with this energy, located at $100 \mathrm{kpc}$, would have a flux equal to the "instrumental" minimum observable flux. A standard deviation of half an order of magnitude was used. This is a somewhat adhoc value; the true distribution in $E$ likely depends on distributions of NS magnetic field strength and spin rate, which are unknown for the burster population. The results are not very sensitive to this spread as along as it is less than two orders of magnitude, where it becomes comparable to the spread in flux due to the range in distances of the bursts.

The relation $\log (N>F) \propto-3 / 2 \log F$ holds for an isotropic distribution of standard candles, where $\log (N>F)$ is the number of bursts with flux greater than $F$. The simulation data are roughly consistent with this distribution for large values of flux, though a slope of -1.44 is the best fit. The flattening of the slope observed in the BATSE data is also seen in the simulation data.

\section{Conclusions}

These results suggest that models of GRBs as comet-NS collisions in an extended Halo are untenable for a wide range of free parameters. While simulation data, with a somewhat unphysical choice for the ejection efficiency of GC stars, roughly meet the distribution in flux of real GRBs, no values of the free parameters allow a fit to the isotropy data. However, this work does assume that the NSs are ejected from the Galactic disk, and are not relics from the initial formation of the Galaxy. Choosing an appropriate distribution of relic NSs may allow a model in agreement with the observations. In addition, replacing the spherical dark matter halo with an oblate one might reduce the clumping toward the Galactic centre.

\section{References}

Johnston, S.: 1994, Monthly Not. Roy. Astr. Soc. 268, 595.

Kulessa, A.S., and Lynden-Bell, D.: 1992, Monthly Not. Roy. Astr. Soc. 255, 105.

Mitrofanov, I.G., and Sagdeev, R.Z.: 1990, Nature 344, 313.

Paczynksi, B.: 1990, Astrophys. J. 348, 485.

Pineault, S., and Duquet, J.-R.: 1993, Monthly Not. Roy. Astr. Soc. 261, 246.

Pineault, S., and Poisson, E.: 1989, Astrophys. J. 347, 1141.

Stern, S.A.: 1990, Publ. Astron. Soc. Pacific 102, 793. 


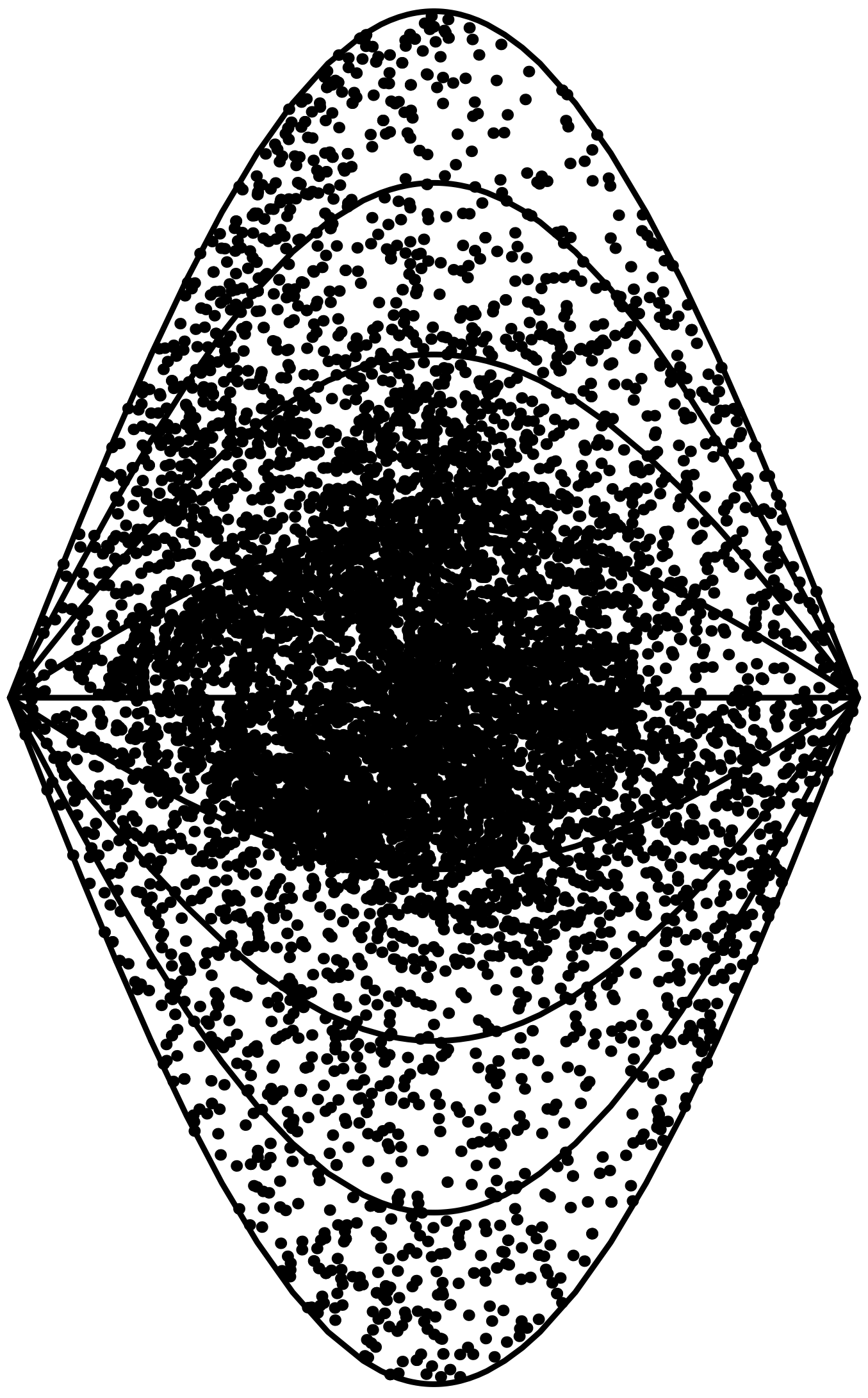

